POS PROCEEDINGS

\title{
Tasks in next 10 years: A personal view on future of searches for beyond the standard model
}

\section{Junji Hisano*}

Department of Physics, Nagoya University, Nagoya 464-8602, Japan

Kavli IPMU (WPI), University of Tokyo, Kashiwa, Chiba 277-8583, Japan

E-mail: hisano@eken.phys.eken.phys.nagoya-u.ac.jp

I was asked to talk about next 10 years in particle physics in this conference, since I joined in the KMI future planing comittee. This is my personal view on searches for physics beyond the standard model at $\mathrm{TeV}$ scale.

KMI International Symposium 2013 on ăAIJQuest for the Origin of Particles and the Universe", 11-13 December, 2013

Nagoya University, Japan

\footnotetext{
* Speaker.
} 


\section{Introduction}

A Higgs boson, which is responsible to origin of mass for elementary particles, has discovered at the LHC experiments [1]. This implies great triumph of the standard model in particle physics. Then, is particle physics over?

We can say "no" for this question obviously. We have several reasons to consider physics beyond the standard model. Non-baryonic dark matter in the universe, tiny masses for neutrinos, dark energy responsible to accelerating expansion of the universe, apparently accusal density fluctuations, and baryon asymmetry in the universe. Those problems are not from aesthetic viewpoints. New theories should be constructed and tested in order to solve them. However, we do know at which energy scale the new physics appears.

In the past 20 years, the naturalness of the Higgs boson mass in the standard model was an important guiding principle in studies of physics beyond the standard model, since it required new physics would appear around TeV scale. The supersymmetric (SUSY) standard model has been the leading candidate. However, searches for new particles, such as SUSY particles, have null results at the LHC experiments. In addition, the observed Higgs boson mass is relatively heavier than prediction of the the supersymmetric standard model at $\mathrm{TeV}$ scale. The naturalness argument may need to modified more or less now. I consider that the supersymmetric standard model is still well-motivated from the the gauge coupling unification and the dark matter. However, even if it is real, is new physics accessible in future experiments?

Thus, in my talk, I pick up several topics from my personal viewpoints. I consider that following three questions are urgent to be solve in particle physics.

- Does deviation of muon $(g-2)$ come from physics beyond the standard model ?

- Is the dark matter WIMPs ?

- Is physics beyond the standard model accessible?

These are related to physics beyond the standard model around or near $\mathrm{TeV}$ scale. I will not cover the neutrino physics though it is going to an interesting new stage to measure the $\mathrm{CP}$ violation and also to determine the mass hierarchy.

In history of particle physics, we have had three approaches to new physics. First is approach with "energy frontier physics". The LHC is working for the purpose. The second one is approach with "precision frontier physics". Precision frontier physics is sometimes related to symmetries and their breaking pattens, and it may have sensitivities to physics at much larger than $\mathrm{TeV}$ scale. The last is "cosmic frontier physics". The astrophysical observations have given hints on "dark" sectors in the particle physics, such as to neutrinos or the dark matter. These three approaches are complimentary to each others. I consider that it is important to search for physics beyond the standard model from every approaches and to combine their results under theoretical studies.

The Kobayashi-Maskawa mechanism was established by conspiracy between energy and frontier physics. The neutrino oscillations were first discovered at the atmospheric and solar neutrino experiments, while the human-made neutrinos from accelerators or reactors are now used for the experiments. I expect history will repeat again in searches for physics beyond the standard model. 
In this proceeding, I omit all figures since my pages are limited. Please see my presentation file on the conference web page if you have interests on them.

\section{Muon (g-2)}

The muon $(g-2)$ measurement is one of the most precise tests in the standard model. The result in the BNL experiment [2] is about (3-4)-sigma deviated from the prediction in the standard model [3].

$$
\left.a_{\mu}\right|_{\exp }-\left.a_{\mu}\right|_{\mathrm{th}} \sim(3 \pm 1) \times 10^{-9}
$$

The first announcement of the BNL result was on 2001. While the standard model prediction and the experimental measurements have been updated since it, this anomaly has not disappeared at all. Thus, it may be a signature of physics beyond the standard model, and it should be tested furthermore.

The new measurement of muon $(g-2)$ are planed at Fermilab and Jparc. The experimental instruments at BNL has been moved to Fermilab, and the measurement will be done with higher statistics. On the other hand, the experiment planed at Jparc is based on a different experimental technique from the the BNL one. Those experiments have comparable sensitives to each others, and they aim to reduce the experimental uncertainty by a factor 4 or 5 .

We also have to refine the standard model prediction furthermore. The muon $(g-2)$ has various contributions even in the standard model. The first is the pure QED contribution, which is calculated up to five-loop level by Kinoshita et al [4]. The second comes from diagrams with the hadronic vacuum polarization inserted. The third is the light-by-light scattering contribution. The last is the electroweak contribution, which is evaluated at two-loop level [5]. The largest uncertainties come from the hadronic vacuum polarization contribution and also the light-by-light scattering contribution. It is important to reduce the uncertainties in those evaluations.

The hadronic vacuum polarization contribution can be evaluated from the $R$ ratio with the dispersion relation. The $R$ ratio is precisely measured from the direct scale experiments such as CMD-2 and SND at Novosibirsk and BES at Beijing, and also measurement of the radiative return processes at the KLOE and $\mathrm{BaBar}$. The current uncertainties of the hadronic vacuum polarization contribution is $\delta a_{\mu} \sim(0.3-0.4) \times 10^{-9}$ [3]. More precise measurements of the radiative return processes is expected at the Belle II experiment with higher statistics.

On the other hand, the light-by-light scattering contribution cannot be extracted from the physical observables. Thus, the evaluation relies on phenomenological models at present. The current prediction of the muon $(g-2)$ is based on the Glasgow consensus for the light-by-light scattering contribution [6], $\left.a_{\mu}\right|_{\mathrm{lbl}}=(1.05 \pm 0.26) \times 10^{-9}$.

In order to reduce the theoretical uncertainty of the standard model prediction for muon $(g-2)$, the light-by-light scattering contribution should be evaluated directly from QCD. An approach in lattice QCD and QED is proposed by Hayakawa et al [7]. This direction looks the most promising.

If the anomaly of the muon $(g-2)$ is real, some new particles are expected to be discovered at the LHC experiments. It is known that the supersymmetric standard model easily explains the anomaly. The supersymmetric standard model has two Higgs doublets, and the slepton-chargino 
one-loop diagram contribution is enhanced by the ratio of the two vacuum expectation values $(\tan \beta)$,

$$
\delta a_{\mu} \simeq 3 \times 10^{-9}\left(\frac{\tan \beta}{10}\right)\left(\frac{M_{\mathrm{SUSY}}}{200 \mathrm{GeV}}\right)^{-2} .
$$

Here, we take the slepton and chargino masses to a common value $M_{\mathrm{SUSY}}$, and the typical value of $\tan \beta$ is from 2 to 60 . In non-supersymmetric models, which do not have enhancement factors, lighter new particles coupled with muon are required to explain the muon $(g-2)$.

Null results in the new particle searches at the LHC experiments give stringent constraints on physics beyond the standard model at $\mathrm{TeV}$ scale, especially in the cases that the models include colored particles. The constrained minimal supersymmetric standard model (CMSSM) is severely constrained from the squark and gluon searches so that the region to explain the anomaly in the muon $(g-2)$ are almost excluded. On the other hand, some supersymmetric models in which mass splitting between squarks and sleptons is larger may be possible. The slepton and chargino searches with the electroweak processes should be more improved to test the anomaly of muon $(g-2)$.

\section{WIMP Dark Matter}

The weakly-interacting massive particles (WIMPs) is candidates for the dark matter in the universe. The WIMPs is well-motivated since the observed dark matter abundance may be well explained under the thermal dark matter production hypothesis. In the early hot universe the WIMPs were thermalized if they have interactions with ordinary particles. When the temperature goes down below $\sim m / 20$ ( $m$ the WIMP mass), the WIMPs are decoupled from the thermal bath, and the WIMP number density (normalized to entropy density) is frozen. The WIMP energy fraction in the current universe is

$$
\Omega h^{2} \sim 0.1\left(\frac{1 \mathrm{pb}}{\langle\sigma v\rangle}\right) \sim 0.1\left(\frac{(20 \mathrm{TeV})^{-2}}{\langle\sigma v\rangle}\right)
$$

where $\langle\sigma v\rangle$ is the thermal averaged annihilation cross section for the WIMPs. When the WIMPs have only "weak" coupling, the thermal dark matter production hypothesis is consistent with new physics around the TeV scale. This coincidence is called as "WIMP miracle", and it motivates us to consider new physics around the TeV scale.

Let us consider the thermal dark matter production hypothesis more precisely. The WIMPs may comes from a neutral component in an $\mathrm{SU}(2)_{L}$ gauge multiplet, similar to neutrinos. For example, Higgsinos and winos in the supersymmetric standard model $\mathrm{SU}(2)_{L}$ doublet and triplet, respectively. Under the thermal dark matter production hypothesis, the observed dark matter abundance is explained when Higgsino and wino masses are about 1 and $3 \mathrm{TeV}$ [8], respectively. When WIMPs comes larger $\mathrm{SU}(2)_{L}$ multiplets, the masses are heavier than them. On the other hand, when the WIMPs are $\mathrm{SU}(2)_{L}$ singlet, the lighter masses are possible. If the WIMP masses are heavier than about $1 \mathrm{TeV}$, it would be quite difficult to find them at the LHC.

The WIMP dark matter are being searched for with the direct and indirect detection methods. They may have sensitivities to the WIMPs even if their masses are above $\mathrm{TeV}$, as will be shown. 
The direct detection of the WIMP dark matter on the earth is to detect the recoiled nuclei with $\mathrm{keV}$ kinetic energy. Ton-scale next-generation detectors may reach to the spin-independent cross section of proton around $10^{-(46-47)} \mathrm{cm}^{2}$. When the WIMPs have only $\mathrm{SU}(2)_{L}$ gauge interactions, Their spin-independent interactions with proton are induced by loop diagrams. It is found that the spin-independent cross section is insensitive to the WIMP masses while the loop factor suppress the cross section [9]. When the dark matter is wino, the spin-independent cross section is about $10^{-47} \mathrm{~cm}^{2}$. Accidental cancellation gives uncertainties of a factor 2 in the cross section. We have to evaluate the QCD correction to the cross section in order to get the reliable prediction.

The indirect searches for the WIMP dark matter are performed in studies of cosmic rays, such gamma rays, positrons, antiprotons, and neutrinos. The searches with gamma rays have a merit that we can identify their sources, compared with cosmic ray positrons and antiprotons. The Fermi satellite observes gamma rays from various directions. The collaboration gives constraints on the WIMP annihilation cross sections from observation of the gamma rays from dwarf Spheroidals. Combining the several constraints from dwarf Spheroidals, it is argued that the thermal dark matter production hypothesis is excluded when the WIMP mass is lighter than $20 \mathrm{GeV}$ [10].

Line gamma rays, whose energy is the WIMP mass, are smoking gun of the WIMP dark matter. The process is induced by loop diagrams so that the cross section is suppressed. This situation is changed when the WIMPs have $\mathrm{SU}(2)_{L}$ gauge charges and their masses are larger than about $1 \mathrm{TeV}$ [11]. The wave functions of WIMP two-bodies non-relativistic system is modified by the $\mathrm{SU}(2)_{L}$ gauge interaction so that the non-relativistic annihilation processes are significantly changed. This phenomena are called as the Sommerfeld enhancement. More interestingly, the WIMP annihilation cross section to two gammas becomes comparable to those for the tree-level processes, such as to a pair of $W$ bosons. Thus, the observation for line gamma rays is sensitive to $\mathrm{SU}(2) L$-interacting WIMPs with masses larger than $1 \mathrm{TeV}$.

The HESS air Cerenkov telescope for gamma rays and the Fermi satellite give constraints on flux of line gamma rays from the galactic center [12]. The HESS telescope gives the constraints with energy larger than $500 \mathrm{GeV}$. Flux of line gamma rays with lower energy is also constrained by Fermi satellite. It is pointed out that if the dark matter density profile around the galactic center is the Navarro-Frenk-While profile, which is motivated by the $N$-bodies simulation, the wino dark matter is almost excluded [13]. On the other hand, the constraint depends on the dark matter density profile. The Burkert dark matter density profile has a core in the center. In the case the thermal dark matter production hypothesis is still viable. Now the Cerenkov Telescope Array (CTA) is planed so that it will detect gamma rays with higher sensitivities. They may get a clue to the WIMP dark matter, or they may get much severer bounds on it.

\section{Is physics beyond the standard model accessible?}

The most serious problem (at least to me) is the energy scale of physics beyond the standard model. While the direct searches for new physics at the LHC experiments are important, I consider that importance of the indirect searches in the precision frontier physics is increasing.

The electric dipole moments (EDMs) of electron and neutron have high sensitivities to physics beyond the standard model. Many models beyond the standard model have new CP violations. Integrating the heavy particles generates the parton-level CP-violating interactions at one- or two- 
loop level. Assuming maximal CP phases, one-loop diagrams for EDMs for quarks or leptons give strong constraint on new physics below the TeV scale,

$$
\frac{d_{e}}{e} \sim \frac{f \bar{f}}{(4 \pi)^{2}} \frac{m_{e}}{M^{2}}=6 \times 10^{-26} \mathrm{~cm} \times f \bar{f}\left(\frac{M}{1 \mathrm{TeV}}\right)^{-2}
$$

and even two-loop diagrams can also constrain new physics around $O(100) \mathrm{GeV}$. Now the improvement on the electron EDM with the molecular EDM measurements is quite active. The latest upperbound on the electron EDM is derived by the ACME collaboration using the polar molecule thorium monoxide, $\left|d_{e} / e\right|<8.7 \times 10^{-29} \mathrm{~cm}[14]$.

Let us consider the supersymmetric standard model. In the model many SUSY breaking parameters introduced there are complex so that the EDMs are predicted. If one-loop diagrams with maximal CP phases generate the electron EDM, the SUSY breaking scale should be larger than $O(10-100) \mathrm{TeV}$. The neutron EDM gives looser constraints on the supersymmetric model if the flavors are conserving and the SUSY breaking parameters have no hierarchical structure among them. However, the neutron EDM also has sensitivities on the quark flavor violation in the squark mass matrices [15], and the finite value of the neutron EDM might be found before the electron EDM. Those measurements are complimentary to identify the model if they are discovered.

The searches for flavor-violating processes are also sensitive to physics beyond the standard model. The muon flavor violating processes, such as $\mu \rightarrow e \gamma$ and $\mu-e$ conversion in nuclei, are predicted in many models, since the lepton-flavor symmetries is not exact in nature due to the neutrino masses. The supersymmetric standard model predicts the charged-lepton flavor violating processes due to the flavor violation in slepton mass matrices, and they may be related to the origin of neutrino masses [16]. While depending on size of the flavor violation, the slepton with about $10 \mathrm{TeV}$ has been constrained by null result of the MEG experiment [17]. The next stage of the MEG experiments and also searches for the $\mu-e$ conversion in nuclei at Mu2e and COMET will probe the higher mass scale.

The quark flavor violation should be tested furthermore. The Kobayashi-Maskawa mechanism is established while there may be rooms for physics beyond the standard model. More precise tests of unitarity of the Cabibbo-Kobayashi-Maskawa matrix will be preformed at the Belle II experiments. If new sources for quark flavor violation appear below the about $10 \mathrm{TeV}$, the sizable deviation may be observed there.

Last, let us discuss the discovered Higgs boson. The Higgs boson might be a new window to physics beyond the standard model. The Higgs boson properties should be precisely measured at the LHC experiments. On the other hand, the properties are constrained from the low-energy experiments.

The flavor-changing couplings of the Higgs boson are automatically suppressed in the standard model. However, if the higher dimensional interactions of the Higgs boson with quarks/leptons are introduced, the Higgs boson may have the flavor-changing couplings. The $\mu$-e transition is severely constrained from the upperbound on $\operatorname{Br}(\mu \rightarrow e \gamma)$. On the other hand, sizable $\tau$ flavor violation may be still allowed. The $\tau-e$ and $\tau$ - $\mu$ couplings with the Higgs boson are constrained from $\operatorname{Br}(\tau \rightarrow \mu / e \gamma)$ while the constraints are comparable to the LHC search for the Higgs boson decays in to $\tau \mu$ and $\tau e$, respectively [18]. From a viewpoint of naturalness in the Yukawa couplings, 
the large flavor violation between $\tau$ and $e$ might be difficult to be sizable. Further improvement on $\operatorname{Br}(\tau \rightarrow \mu / e \gamma)$ will give constraints on the flavor-changing higher dimensional interaction.

The CP-violating couplings of the Higgs boson with other standard model particles are also derived with the higher dimensional interactions. They are constrained to the electron and neutron EDMs. The Barr-Zee diagrams contribute to the EDMs at two-loop level [19]. The diagrams include a CP violating $\gamma^{*} \gamma h$ coupling, which is generated by loops of charged fermions coupled with the Higgs boson. While the diagrams are at two-loop level, the severe bounds on the neutron and, especially, electron EDMs can give the CP violating couplings.

\section{Summary}

We have no doubt that the LHC experiments may still have chances to discover new physics. The muon $(g-2)$ anomaly and the WIMP dark matter should be tested there. On the other hand, our abilities to probe physics beyond the standard model should be enhanced as possible. I discuss my view for next ten years in particle physics from viewpoints of $\mathrm{TeV}$-scale physics. New physics searches should be pursued from all three approaches, energy, precision, and cosmic frontiers. And, theoretical studies are also required in order to enhance the abilities of new physics searches, and also to correctly interpret the signals if they are discovered.

\section{References}

[1] G. Aad et al. [ATLAS Collaboration], Phys. Lett. B 716 (2012) 1 [arXiv:1207.7214 [hep-ex]]; S. Chatrchyan et al. [CMS Collaboration], Phys. Lett. B 716, 30 (2012) [arXiv:1207.7235 [hep-ex]].

[2] G. W. Bennett et al. [Muon G-2 Collaboration], Phys. Rev. D 73 (2006) 072003 [hep-ex/0602035].

[3] K. Hagiwara, R. Liao, A. D. Martin, D. Nomura and T. Teubner, J. Phys. G 38 (2011) 085003 [arXiv:1105.3149 [hep-ph]]; T. Teubner, K. Hagiwara, R. Liao, A. D. Martin and D. Nomura, Chin. Phys. C 34 (2010) 728 [arXiv:1001.5401 [hep-ph]]; M. Benayoun, P. David, L. DelBuono and F. Jegerlehner, Eur. Phys. J. C 72 (2012) 1848 [arXiv:1106.1315 [hep-ph]]; . Davier, A. Hoecker, B. Malaescu and Z. Zhang, Eur. Phys. J. C 71 (2011) 1515 [Erratum-ibid. C 72 (2012) 1874] [arXiv:1010.4180 [hep-ph]].

[4] T. Aoyama, M. Hayakawa, T. Kinoshita and M. Nio, Phys. Rev. Lett. 109 (2012) 111808 [arXiv:1205.5370 [hep-ph]].

[5] A. Czarnecki, W. J. Marciano and A. Vainshtein, Phys. Rev. D 67 (2003) 073006 [Erratum-ibid. D 73 (2006) 119901] [hep-ph/0212229].

[6] J. Prades, E. de Rafael and A. Vainshtein, (Advanced series on directions in high energy physics. 20) [arXiv:0901.0306 [hep-ph]].

[7] S. Chowdhury, T. Blum, T. Izubuchi, M. Hayakawa, N. Yamada and T. Yamazaki, PoS LATTICE 2008 (2008) 251.

[8] J. Hisano, S. Matsumoto, M. Nagai, O. Saito and M. Senami, Phys. Lett. B 646 (2007) 34 [hep-ph/0610249].

[9] J. Hisano, S. Matsumoto, M. M. Nojiri and O. Saito, Phys. Rev. D 71 (2005) 015007 [hep-ph/0407168]; J. Hisano, K. Ishiwata and N. Nagata, Phys. Lett. B 690 (2010) 311 [arXiv:1004.4090 [hep-ph]]; J. Hisano, K. Ishiwata, N. Nagata and T. Takesako, JHEP 1107 (2011) 005 [arXiv:1104.0228 [hep-ph]]. 
[10] M. Ackermann et al. [Fermi-LAT Collaboration], Phys. Rev. Lett. 107 (2011) 241302 [arXiv:1108.3546 [astro-ph.HE]].

[11] J. Hisano, S. Matsumoto and M. M. Nojiri, Phys. Rev. Lett. 92 (2004) 031303 [hep-ph/0307216]; J. Hisano, S. .Matsumoto, M. M. Nojiri and O. Saito, Phys. Rev. D 71 (2005) 063528 [hep-ph/0412403].

[12] A. Abramowski et al. [H.E.S.S. Collaboration], Phys. Rev. Lett. 110 (2013) 041301 [arXiv:1301.1173 [astro-ph.HE]].

[13] T. Cohen, M. Lisanti, A. Pierce and T. R. Slatyer, JCAP 1310 (2013) 061 [arXiv:1307.4082], J. Fan and M. Reece, JHEP 1310 (2013) 124 [arXiv:1307.4400 [hep-ph]].

[14] J. Baron et al. [ACME Collaboration], Science 343 (2014) 6168, 269 [arXiv:1310.7534 [physics.atom-ph]].

[15] J. Hisano, M. Nagai and P. Paradisi, Phys. Rev. D 80 (2009) 095014 [arXiv:0812.4283 [hep-ph]].

[16] F. Borzumati and A. Masiero, Phys. Rev. Lett. 57 (1986) 961; J. Hisano, T. Moroi, K. Tobe, M. Yamaguchi and T. Yanagida, Phys. Lett. B 357 (1995) 579 [hep-ph/9501407]; J. Hisano, T. Moroi, K. Tobe and M. Yamaguchi, Phys. Rev. D 53 (1996) 2442 [hep-ph/9510309].

[17] J. Hisano, M. Nagai, P. Paradisi and Y. Shimizu, JHEP 0912 (2009) 030 [arXiv:0904.2080 [hep-ph]]; K. Fuyuto, J. Hisano, N. Nagata and K. Tsumura, JHEP 1312 (2013) 010 [arXiv:1308.6493 [hep-ph]].

[18] R. Harnik, J. Kopp and J. Zupan, JHEP 1303 (2013) 026 [arXiv:1209.1397 [hep-ph]].

[19] J. Brod, U. Haisch and J. Zupan, JHEP 1311 (2013) 180 [arXiv:1310.1385 [hep-ph], arXiv:1310.1385]. 